\title{
LA INFANCIA ENTRE LO PÚBLICO Y LO PRIVADO Una revisión al valor de la familia en Rawls
}

\author{
CHILDHOOD BETWEEN THE PUBLIC AND THE PRIVATE \\ $A$ review of the value of family in Rawls
}

\author{
María José Jara Leiva*
}

\begin{abstract}
RESUMEN: El tema de la interferencia estatal en las funciones parentales nos conduce a la dicotomía entre lo público y lo privado, propia de teorías liberales y ampliamente criticada por feministas. El presente artículo reflexiona en torno a los planteamientos, críticas y reconsideraciones de las ideas de Rawls acerca del valor de la familia y su relación con el Estado. Se sostiene que, para el autor, la familia es una institución ambivalente, y que eso se traduce en contradicciones en su postura, la que -en todo caso- no presta atención a la situación de los niños y las niñas como personas actuales dentro de esa esfera. Sin perjuicio de eso, la revisión de algunas de sus ideas permite afirmar que el ámbito familiar, y particularmente las situaciones que involucran a los niños y las niñas, no deben ser herméticos ante los principios de justicia, siendo los derechos humanos un discurso válido y necesario en el campo de las relaciones intersubjetivas.
\end{abstract}

ABSTRACT: The issue of the State interference in parental role leads us to the dichotomy between the public and the private, typical of liberal theories and widely criticized by feminists. This paper focuses on the approaches, criticisms and reconsiderations of Rawls' thoughts about the value of the family and its relationship with the State. It is argued that for the author the family is an ambivalent institution, and that this explains contradictions in his theory, which, in any case, does not pay attention to the situation of present children within this sphere. Regardless of the foregoing, the revision of some of his ideas allows us to assert that the family ambit, and specially situations involving children, should not remain inscrutable for the principles of justice. Human rights are a valid and necessary discourse in the field of intersubjective relations.

PALABRAS CLAVE: Rawls, familia, autonomía familiar, principios de justicia, desventajas, niños y niñas.

KEY WORDS: Rawls, family, family autonomy, principles of justice, disadvantages, children.

Fecha de recepción: 15/10/2021

Fecha de aceptación: 01/11/2021

doi: https://doi.org/10.20318/universitas.2022.6573

\footnotetext{
* Doctoranda en Derecho de la Universidad Diego Portales, Máster en Estudios Avanzados en Derechos Humanos de la Universidad Carlos III de Madrid. E-mail: mariajose.jara.l@gmail.com
} 


\section{1.- INTRODUCCIÓN}

Desde hace algunos años, los temas de la crianza de los hijos y las hijas, la injerencia del Estado en la vida familiar, y la relación entre la responsabilidad parental y la autonomía progresiva de los niños y las niñas, han motivado debates y tensiones en España y Latinoamérica. Una muestra de lo anterior es lo recientemente ocurrido en Chile. En el 2021, el Tribunal Constitucional chileno acogió un requerimiento de inconstitucionalidad presentado en contra de algunos de los preceptos del proyecto de ley que crea el sistema de garantías de los derechos de la niñez (tras varios años de tramitación en el Congreso $)^{1}$

Lo que tenían en común las disposiciones impugnadas era referirse $-y$ supuestamente afectar- al derecho preferente de los padres de educar a sus hijos, reconocido en la Constitución chilena ${ }^{2}$, y materializar algunos de los efectos de la autonomía progresiva de los niños y las niñas en distintos ámbitos.

En su voto de mayoría, aunque el tribunal reconoce que los niños y las niñas son titulares de derechos fundamentales, afirma que estos no tienen relevancia en el seno de la familia. Para el tribunal, la familia es una esfera de relaciones que no son regulables jurídicamente. Esto no quiere decir que no posea un sistema de reglas, si no que estas son libremente establecidas por el padre y la madre, guiados por su deber moral, para desplegar su autoridad y juicio en el bien de su progenie ${ }^{3}$. Vale la pena citar parte de su considerando $7^{\circ}$ :

bajo la justificación de estar velando por el interés superior del niño se avanza[sic] (con sutileza sólo en apariencia) hacia la sustitución del rol tutelar de los padres por el del Estado a través del otorgamiento de derechos a hijos menores de edad susceptibles de ser invocados como límites a la autoridad de su madre o padre. El hermoso deber de los padres de familia de educar a sus hijos está siendo reducido, por medio de la juridificación estatal de la vinculación filial, a un inferior deber de respeto a una cierta interpretación de autonomía progresiva dictada por terceros (el Estado en sus distintas facetas) (...).

El razonamiento judicial aludido nos invita a hacernos algunas preguntas respecto de los derechos de los niños y las niñas en el seno de una familia, y el rol que le cabe al Estado frente a los problemas

${ }^{1}$ Tribunal Constitucional de la República de Chile. Sentencia de 26 de julio de 2021, Rol No 11.315/11.317-21-CPT (acumuladas). Cabe señalar que esta ley constituye una deuda histórica para el país, siendo a la fecha el único de Latinoamérica, junto con Panamá, que no cuenta con una.

2 "Los padres tienen el derecho preferente y el deber de educar a sus hijos. Corresponderá al Estado otorgar especial protección al ejercicio de este derecho". Constitución Política de la República de Chile, artículo $19 \mathrm{~N}^{\circ} 10$, inciso $2^{\circ}$.

3 Tribunal Constitucional de la República de Chile. Sentencia de 26 de julio de 2021, Rol No $11.315 / 11.317-21-\mathrm{CPT}$ (acumuladas), considerando $4^{\circ}, 6^{\circ}, 7^{\circ}, 9^{\circ}, 10^{\circ} \mathrm{y} 11^{\circ}$. 
que en ella se suscitan. En una sociedad democrática, ¿puede y debe el Estado intervenir en la familia en favor de los más desventajados en sus relaciones internas, y específicamente los niños y las niñas? De hacerlo, ¿estaría afectando la autonomía familiar? En definitiva, ¿son las paredes del hogar un muro infranqueable para la justicia y el discurso de los derechos?

Estas preguntas conducen a un debate de larga data respecto de la naturaleza pública o privada de las relaciones familiares y el rol del Estado ante las desventajas de ciertas personas en esta esfera, como los niños y las niñas. En este, la teoría de la justicia de Rawls ha sido ampliamente discutida. Considero que analizar algunos aspectos de la teoría de Rawls en la materia, examinando sus críticas, así como la defensa de sus ideas, nos pueden dar importantes luces acerca de cómo resolver, en un marco de democracia y pluralismo, los problemas jurídicos que afectan a niños y niñas en el ámbito privado debido a su posición dentro de la familia.

En este contexto, el presente artículo busca analizar algunas de las ideas centrales de Rawls en cuanto a la familia, especialmente el valor que le otorga a esta y el lugar que le asigna a las relaciones familiares, a partir de la revisión de sus planteamientos y críticas. Se propondrá que, aunque su postura presenta ambivalencias y contradicciones, es posible arribar a una interpretación de sus ideas que, a la luz de los derechos de los niños y las niñas, y en virtud de su posición de sujetos de derechos, nos permita atender a su situación de desventaja en la relación familiar, sin por eso alterar la autonomía de las relaciones en esa esfera.

Para lo anterior, en primer lugar, se hace referencia a las críticas feministas a la dicotomía público/privado sostenida, en general, por las teorías liberales. Luego, se plantea que la familia ha de ser objeto de la justicia por el hecho de que puede llegar a ser una potencial fuente de desventaja (o de ventajas inmerecidas), y también una fuente de opresión de los niños y las niñas, como lo han planteado especialmente las teorías liberacionistas de la infancia, todo lo cual subraya la relevancia jurídica de aquella institución social y sus relaciones. Posteriormente, se enuncian algunas de las razones que se han esgrimido para justificar, pese a las paradojas que esta presenta, la existencia de la familia, que tienen que ver con las funciones que ella cumple en la sociedad. Después, se describe la tensión existente en Rawls acerca de si la familia es parte de la estructura básica de la sociedad o bien una agrupación voluntaria, y sus implicancias. Por último, se plantea una respuesta a la pregunta de si lo doméstico, y particularmente las relaciones intersubjetivas que pueden llegar a afectar a los niños y las niñas como grupo, puede ser considerado, desde la teoría de Rawls, como un ámbito impenetrable para la justicia. Finalmente, se presentan algunas conclusiones. 


\section{2.- CRÍTICAS FEMINISTAS A LA DICOTOMÍA PÚBLICO/PRIVADO}

Durante el siglo $X X$, especialmente en su segunda mitad, el reconocimiento de derechos que antes se encontraban reservados para los hombres y personas adultas, se ha ido ampliando, a nivel formal, hasta alcanzar a otros grupos, como las mujeres y los niños y las niñas. No obstante, diversas corrientes feministas han coincidido en que la consecución de igualdad de derechos de las personas no ha logrado eliminar la desigualdad que permanece latente tras las estructuras sociales en las que se relacionan. Un elemento fundamental de esta desigualdad subyacente ha sido la tradicional distinción liberal entre los mundos público y privado, que admite y perpetúa en esta última esfera relaciones de dominación ${ }^{4}$. En otras palabras, tras la igualdad formal y racionalidad universal, las desigualdades en la esfera de lo privado han permanecido "ocultas bajo la artificial unidad de lo público" 5 .

En lo anterior, un espacio clave es lo que sucede en el espacio doméstico-familiar. Tradicionalmente, desde el punto de vista liberal, se ha entendido que la vida familiar, donde ha de desarrollarse la crianza de los niños y las niñas, pertenece a una esfera de intimidad de las personas. Constituye un refugio íntimo frente a la coerción estatal y las presiones sociales, donde sus integrantes pueden llevar a cabo sus planes de vida y desarrollar relaciones de afectividad en condiciones de libertad y privacidad ${ }^{6}$. Más aún, constituye un valor para el liberalismo que los progenitores críen a su progenie en este ambiente íntimo y libre de injerencias externas, puesto que forma parte del pluralismo y la diversidad de formas de vida que deben ser respetados como consecuencia de la autonomía y libertad de las personas. En este sentido, cada familia es expresión de esa libertad, y el niño o la niña crecerá conforme a las creencias y valores que les transmitan su padre y madre ${ }^{7}$. Por eso, las relaciones que se desarrollen en este espacio - así entendido- deben permanecer, salvo situaciones muy excepcionales, inmunes a interferencias del Estado y terceros.

No obstante, como explica Udi, para el liberalismo político la familia ha sido tanto un valor como un problema. Por un lado, como ya

\footnotetext{
${ }^{4}$ Isabel Turégano, "La dicotomía público/privado y el liberalismo político de J. Rawls" (2001), Doxa, 319-323.

5 Íbid, 322; Carole Pateman, El contrato sexual (1 ed, Anthropos, 1998), 10-11; Susan Moller Okin, Justice, gender and the family (Basic Books Inc., Nueva York, 1989), 3-6.

6 Isabel Turégano, "La dicotomía público/privado y el liberalismo político de J. Rawls" 324-325. David Archard, Children. Rights and childhood, ( $2^{\circ}$ ed, Routledge, Oxon, 2004), 153-159.

7 David Archard, Children, family and the State (Routledge revivals, Londres y Nueva York, 2018), 74-75.
} 
se describió, se presenta como un refugio de intimidad de las personas. Además, es valiosa para la sociedad en su conjunto, pues cumple funciones como la reproducción de los modos de vida y la socialización. Pero, por otro, las características de la familia, sobre todo en su patrón tradicional (marcada por la jerarquía y la subordinación al pater), puede entrar en conflicto con ideas que son -0, al menos, deberían ser- esenciales para el liberalismo político, tales como la igualdad de oportunidades, y los derechos de las mujeres y de los niños y las niñas ${ }^{8}$.

En este sentido, autores de los orígenes del contractualismo liberal, particularmente Hobbes y Locke, abordaron esta tensión y reflexionaron en torno a la idea de la familia y la autoridad parental (en manifiesta oposición al patriarcalismo de Filmer ${ }^{9}$ ). No obstante, existieron entre ambos autores importantes diferencias respecto de las características y los límites del poder de los progenitores sobre su progenie. Como hace ver Archard, El Leviatán les entregaba a los padres un dominio absoluto sobre su progenie ${ }^{10}$. En cambio, Locke más liberal- planteaba que el poder parental se justificaba en la incapacidad intrínseca de la condición de la niñez, de manera que no es un poder absoluto, sino que, al estar dirigido a procurar el interés de sus hijos e hijas (que no puede ser satisfecho por ellos mismos dada su incapacidad), se ve limitado por este interés ${ }^{11}$. Sin embargo, Locke no se ocupó de trazar los límites de esa autoridad, probablemente porque asumía que el afecto natural de los progenitores hacia su descendencia les dotaría de una disposición innata para actuar según lo que fuera mejor para el interés de su prole ${ }^{12}$. Además, Locke, en un esfuerzo por separar Estado y familia, descartó, como sí planteaba Hobbes, que existiera una vinculación entre el poder político y el parental, y que este último sea una especie de base del primero, ya que se trataría de distintos tipos de poder $^{13}$.

\footnotetext{
8 Juliana Udi, "El valor de la familia en la teoría de la justicia de Rawls" (2017), No 47, Isonomía, 110.

9 Robert Filmer, en su libro El Patriarca, defiende el poder absoluto del monarca, asimilando el poder natural que este tiene sobre sus súbditos con el del padre de familia sobre sus hijos e hijas, respecto de los cuales tiene pleno e ilimitado dominio. Robert Filmer, Pathriarca and other political works of Sir Robert Filmer (Basil Blackwell Oxford, Oxford, 1949), 63.

10 David Archard, Children. Rights and childhood, 8.

11 John Locke, Segundo tratado sobre el gobierno civil. Un ensayo acerca del verdadero origen, alcance y fin del Gobierno Civil (traducido por: Mellizo, C., Tecnos, Madrid, 2006), 61, 63, 66 y 69.

12 "Dios ha tejido, entre los principios de la naturaleza humana, un sentimiento de ternura para con los hijos propios; y este sentimiento es tan intenso, que apenas es de temer que los padres hagan uso de este poder suyo con extremado rigor. Si hay algún exceso, éste no será casi nunca por excesiva severidad, pues la tendencia natural tirará hacia el extremo opuesto". Íbid, 69-70.

13 John Locke, Ensayo sobre el gobierno civil ( $1^{\circ}$ ed. electrónica, Letras para volar, Guadalajara, 2015), párr. 169-174. Pateman rebate lo anterior, planteando que el poder político tiene su génesis en el poder paternal. En efecto, la separación drástica
} 
Las críticas feministas al contractualismo, aunque no cuestionan necesariamente el carácter privado de la esfera doméstica, sí han rebatido su visión estrictamente dicotómica. Autoras como Okin y Pateman plantean que esa separación provoca que se invisibilice su interrelación con la vida social y política, pues las desigualdades que se dan en su seno inciden también en una desigual posición en la sociedad; y que se toleren y encubran violaciones a los derechos, particularmente de los más desaventajados en su interior ${ }^{14}$. Sostienen también que, concebir a la familia como una esfera impenetrable para los principios políticos y de justicia, iría contra el propio proyecto liberal de reconocer derechos y libertades a todas las personas. Por eso, han exigido a la teoría de la justicia y la filosofía política ocuparse, también, de lo privado, específicamente de la estructura familiar ${ }^{15}$.

Rawls, como liberal, intentó hacerse cargo de algunas de estas críticas. En su teoría, revisó la separación radical entre lo público y lo privado, propia del contractualismo clásico, planteando que son espacios interconectados y que a ambos les son aplicables los principios de justicia ${ }^{16}$. Sus esfuerzos, sin embargo, no han permanecido libres de nuevos reproches. Las principales críticas feministas a Rawls en torno a la idea de la familia han apuntado a tres aspectos centrales ${ }^{17}$ : i) la idea rawlsiana de que las personas que participan en la posición original no son individuos aislados, sino cabezas de familia, lo cual parece dar por hecho que el pater familae opera bajo los principios de justicia como imparcialidad, pues buscará procurar el bienestar de su prole; ii) la falta de referencia al género como característica individual que ha de quedar oculta bajo el velo de ignorancia en la posición original ${ }^{18}$; y iii) la falta de preocupación por la justicia en la estructura familiar, pese al rol fundamental que Rawls

planteada desde el liberalismo tiene como consecuencia la separación de la esfera política de la familiar-privada. Carole Pateman, El contrato sexual, 76-79, 129-130.

14 Isabel Turégano, "La dicotomía público/privado y el liberalismo político de J. Rawls", 325; Carole Pateman, El contrato sexual, 23.

15 Juliana Udi, "El valor de la familia en la teoría de la justicia de Rawls", 110; Isabel Turégano, "La dicotomía público/privado y el liberalismo político de J. Rawls", 326; Susan Moller Okin, Justice, gender and the family; Carole Pateman, El contrato sexual.

16 John Rawls, Liberalismo político (traducido por: Madero, S., Fondo de Cultura Económica, México DF, 1995), 779, 787-792; Isabel Turégano, "La dicotomía público/privado y el liberalismo político de J. Rawls", 320, 335-337; Martha Nussbaum, Las mujeres y el desarrollo humano (traducido por: Bernet, R., $2^{\circ}$ ed., Herder, Barcelona, 2017), 356-357. Esta afirmación de Rawls plantea contradicciones, como se verá.

${ }^{17}$ Isabel Turégano, "La dicotomía público/privado y el liberalismo político de J.

Rawls", nota al pie No 29

${ }^{18}$ Esta idea es revisada y corregida por Rawls en el Liberalismo Político. En esta obra, el género pasa a ser un aspecto que sería relevante de no ser conocido por quienes están en la posición original. John Rawls, Liberalismo político, 47. 
le reconoce en la educación moral y la formación del sentido de justicia ${ }^{19}$.

Todo esto parece indicar que Rawls "parte de la presunción de que la familia tradicional es justa, a pesar de que, de hecho, esté en contradicción con las principales exigencias de la justicia como equidad"20. De hecho, el mismo autor parece reconocer que esa última afirmación está contenida en su obra Teoría de la Justicia, al indicar que en ella había dejado de lado ciertos temas importantes, entre ellos, indica, "la justicia de la familia y en el seno de la familia, aunque supongo que, de alguna forma, la familia es una institución justa"21. No obstante, advierte Rawls, el no abordar directamente la cuestión de la familia no quiere decir que la concepción de justicia que elabora a partir de otros problemas clásicos no pueda ser extrapolable para resolver los conflictos que se susciten en la esfera familiar ${ }^{22}$. Esta idea, sin embargo, presenta algunas contradicciones en sus obras, como se verá.

\section{3.- FAMILIA COMO POTENCIAL FUENTE DE DESVENTAJAS Y DE OPRESIÓN PARA NIÑOS Y NIÑAS}

Según la historiografía de la familia, si bien esta institución ha estado presente bajo distintas formas en todas las sociedades, en la época de la industrialización se consolidó en Europa un modelo particular de familia: la burguesa tradicional. Como explica Valpuesta, se trata de un ideal basado en la relación conyugal y tendencialmente estable, que se organiza en torno al reparto de papeles que sitúan al marido en el trabajo y a la mujer en el hogar. Mientras, a los hijos y las hijas les corresponde dedicarse exclusivamente a su formación y educación, bajo la mirada atenta de sus progenitores; aunque solo los legítimos, puesto que la filiación extramatrimonial era excluida de este modelo ${ }^{23}$. Según la autora, este modelo refuerza la escisión entre lo privado, como parte de la esfera doméstica en la que se desarrolla la vida familiar (a la cual estarían relegados la mujer y los niños y las niñas, bajo el gobierno del pater), y lo público, donde se desarrollan las actividades productivas, políticas y sociales (en el que se desenvuelve el marido-padre) ${ }^{24}$.

19 Isabel Turégano, "La dicotomía público/privado y el liberalismo político de $\mathrm{J}$. Rawls", nota al pie No 29; Susan Moller Okin, Justice, gender and the family, 26-32; Carole Pateman, El contrato sexual, 61-63.

${ }^{20}$ Isabel Turégano, "La dicotomía público/privado y el liberalismo político de J. Rawls" nota al pie $\mathrm{N}^{\circ} 29$.

21 John Rawls, Liberalismo político, 22.

22 Íbid, 23.

23 Rosario Valpuesta, La disciplina constitucional de la familia en la experiencia europea (Tirant lo Blanch, Valencia, 2012), 102.

24 Íbid, 75-77. 
Las desventajas producidas en contra de los grupos dominados al interior de la familia (especialmente la tradicional), mujeres, niños y niñas, han sido abordadas desde diversas perspectivas.

Por ejemplo, para Okin, la base de la desigualdad social que presentan las mujeres en relación con los hombres deviene de una inequitativa distribución de las cargas y los roles en la familia, que permanece oculta tras la clásica separación de lo privado (o espacio doméstico) y lo público (donde se desarrollan la política y el mercado) ${ }^{25}$.

Nussbaum, en su libro Las Mujeres y el desarrollo humano, reconoce que, si bien pueden existir amor y cuidado en la familia, "sería difícil negar que la familia ha sido, si no el mayor, al menos uno de los más importantes sitios de opresión de las mujeres"26. Además, teniendo en cuenta su función en la reproducción social y el tipo de relaciones que se producen en su interior, la familia termina siendo para las futuras generaciones una escuela para la desigualdad, mediante los patrones culturales aprendidos en la crianza 27.

De otro lado, existe también una desigualdad etaria, pues la posición que ocupan los integrantes de la familia varía según si son personas adultas o niños y niñas, pues serían las primeras quienes determinan el mejor interés de los segundos. Desde el punto de vista filosófico, la restricción de la autonomía de los niños y las niñas en el seno de la familia bajo el poder del padre -y posteriormente también de la madre- se funda en la concepción moderna de la infancia, propia de las sociedades liberales. Esta entiende que los niños y las niñas tienen una naturaleza y características claramente separadas y distintas de aquellas que poseen las personas adultas, lo que exige que sean mantenidos en mundos apartados, determinando roles, responsabilidades y modos de vida diferenciados. Además, son vistos como seres incompletos, que se encuentran transitando un estadio de devenir inevitable hacia la meta de la adultez. Ese rasgo explicaría y justificaría su dependencia y subordinación hacia el mundo adulto, especialmente sus padres y madres, quienes serán los que determinarán y procurarán satisfacer - según su propio criterio- sus necesidades e intereses ${ }^{28}$.

En virtud de esta concepción se ha asumido que los niños y las niñas, por su falta de madurez, son, de pleno derecho, incompetentes básicos, negándoles la posibilidad de agencia. Sobre tal presunción, las personas adultas deben intervenir en sus decisiones, acciones 0 comportamientos mediante el paternalismo jurídico. Así, para Garzón Valdés, "en el caso de los niños, el ejercicio de su autonomía está

\footnotetext{
${ }^{25}$ Susan Moller Okin, Justice, gender and the family.

26 Martha Nussbaum, Las mujeres y el desarrollo humano, 321.

27 Íbid, 321-323.

28 David Archard, Children. Rights and childhood, 37-50.
} 
condicionado por una situación de radical vulnerabilidad"29, dada su falta de racionalidad y dependencia, lo que justificaría el paternalismo respecto de ellos.

En relación a la posición de desigualdad basada en el criterio de la edad, liberacionistas como Farson y Holt buscaron librar a los niños y las niñas de las situaciones de opresión y dominación a la que la habrían sometido las personas adultas, especialmente a través de la familia y la escuela. Planteaban que, mediante su relegación a estos espacios, se segrega la niñez del mundo adulto. Sostuvieron que las razones para su tratamiento diferenciado se basan solo en la edad, lo que constituye un criterio arbitrario y por ende discriminatorio. Por eso, abogaron por extender a todos los niños y las niñas los mismos derechos que se les reconocen a las personas adultas, con igual capacidad para ejercerlos ${ }^{30}$ y, como consecuencia, liberarlos del yugo familiar.

En la actualidad encontramos distintas variedades de formas de familia, que responden a los distintos contextos sociales, legales y económicos, así como a la diversidad cultural. Familias nucleares, extendidas, biparentales, monoparentales, cohabitantes, concubinatos, familias reconstituidas, entre otras, que cada vez están más reconocidas socialmente, impiden hablar con propiedad de familia, singular, aludiendo a un modelo universal. Sobre su organización interna, Giddens afirma que, en términos generales, el poder patriarcal ha ido perdiendo fuerza a lo largo del siglo XX, producto de fenómenos como la incorporación de la mujer en el mercado laboral y el predominio de los vínculos basados en la afectividad y la corresponsabilidad ${ }^{31}$.

No obstante, la distribución desigual de tareas y la violencia doméstica siguen estando presentes en nuestras sociedades, perpetuando los patrones de género tradicionales. La desigualdad en las relaciones familiares se suele basar en la idea implícita de que a los hombres y a las mujeres les corresponde responsabilidad en distintas esferas: hombres proveedores y mujer encargada del cuidado ${ }^{32}$. Por su parte, la concepción moderna de la infancia sigue estando presente. Como indica Archard, "en nuestra cultura, esta mirada determina el lugar apropiado del niño como aquel que no puede disfrutar de los derechos y responsabilidades de los adultos"33. Parte de esto es el

${ }^{29}$ Ernesto Garzón, "Desde la 'Modesta Propuesta' de J. Swift hasta Las 'Casas de Engorde': Algunas consideraciones acerca de los derechos de los niños" (1994), Doxa, 15, 737.

30 David Archard, Children. Rights and childhood, 70-74. John Holt, Escape from childhood. The needs and rights of children (Penguin Books, Hardmondsworth, 1975), 21,32 , 59-60 y 88; Richard Farson, Birthrights, (Macmillan Publishing, Nueva York, 1974), 27.

${ }^{31}$ Anthony Giddens, Sociología (traducido por: Muñoz, F., $6^{\circ}$ ed., Alianza Editorial, Madrid, 2014), 368-370.

32 Íbid, 374-378.

33 David Archard, Children. Rights and childhood, 50 [traducción de la autora]. 
mismo concepto del interés superior del niño, reconocido en el artículo 3.1 de la Convención sobre los Derechos del Niño, tras el cual subyace la idea de una tercera persona (adulta, y generalmente su padre $\mathrm{y} / \mathrm{o}^{34}$ madre) tomará las decisiones por ellos hasta que alcance la mayoría de edad. Y es que la familia y sus relaciones no están libres de constantes resistencias, tensiones y contradicciones ${ }^{35}$.

La discusión acerca de la condición de los niños y las niñas como sujetos de derechos positivos quedó en gran parte superada con la masiva ratificación de la Convención sobre los Derechos del Niño. Desde el punto de vista de los derechos morales, las teorías de los derechos se han encontrado con mayores problemas para fundamentar su titularidad ${ }^{36}$. Pero, siguiendo a MacCormick, esa dificultad no debe poner en duda la afirmación de que los niños y las niñas tienen derechos, puesto que ello carecería de toda sensatez ${ }^{37}$.

Por su parte, Rawls, preocupado por la situación de desventaja de las personas, propone como principios de justicia el de igual repartición de derechos y bienes básicos, y los de diferencia e igualdad de oportunidades. Estos últimos buscan producir beneficios compensadores para todas las personas, especialmente las más desaventajadas ${ }^{38}$. En particular, una aplicación consecuente del principio de igualdad de oportunidades exige considerar a las personas independientemente de la influencia de su posición social ${ }^{39}$. Sin embargo, tenemos que, "[i]ncluso en una sociedad bien ordenada que satisfaga los dos principios de justicia, la familia puede ser una barrera para la igualdad de oportunidades entre las personas"40.

Según Rawls, dada la fuerte relevancia que tiene la institución de la familia en las personas, especialmente durante sus primeros años de vida, en el desarrollo de las capacidades y el modelamiento de las aspiraciones personales, esta puede favorecer o bien obstaculizar a sus integrantes la posibilidad de acceder a la igualdad de oportunidades. Así, más allá de los dotes naturales, la suerte, el esfuerzo o el mérito individual, el origen familiar puede terminar condicionando la posibilidad de acceder a las mejores posiciones en la sociedad, y con

\footnotetext{
${ }^{34}$ Íbid, 62.

${ }^{35}$ Respecto de la sociedad chilena: Ximena Valdés, La vida en común. Familia y vida privada en Chile y el medio rural en la segunda mitad del siglo XX ( $1^{\circ}$ ed., Lom Ediciones, Santiago, 2007), 52-53 y 379-388.

36 En términos generales, las explicaciones se han desenvuelto entre las teorías de la voluntad y del interés. En español, ver: Isabel Fanlo, [comp.], Derecho de los niños. Una contribución teórica (Biblioteca de Ética, Filosofía del Derecho y Política, México DF, 2004).

37 Neil MacCormick, "Los derechos de los niños: un test para las teorías de los derechos", en: Isabel Fanlo [comp.], Derecho de los niños. Una contribución teórica (Biblioteca de Ética, Filosofía del Derecho y Política, México DF, 2004), 61.

38 John Rawls, Teoría de la justicia (traducido por: González, M., Fondo de cultura económica, México DF, 1997), 62-118.

39 Íbid, 461.

40 Íbid, 279.
} 
ello, ser un obstáculo para el principio de igualdad de oportunidades ${ }^{41}$. $Y$, dado que la familia es en sí inmerecida (en el sentido de que no obedece al mérito), las ventajas y privilegios que encontramos en nuestra cuna deberían ser sometidas al principio de diferencia.

Siendo así, desde una perspectiva liberal y cimentada en el contractualismo, ¿cómo se justifica la familia, habida cuenta de la subordinación no consensuada de ciertos grupos bajo una autoridad, y la desigualdad de oportunidades que genera? ¿No sería, entonces, más conveniente desde esta perspectiva abolir la familia? ${ }^{42}$.

\section{4.- ENTONCES, ¿QUÉ HACEMOS CON LA FAMILIA?}

Rawls se formuló esa última pregunta, y en distintas partes de sus obras da razones para justificar la existencia de la familia, sobre la base de que ella nos ofrece más luces que sombras. En la justicia como imparcialidad, la familia ocupa un rol trascendental para la reproducción y la estabilidad de una sociedad justa ${ }^{43}$. Por su parte, sus efectos no deseados, indica, pueden ser aplacados con una aplicación adecuada de los principios de fraternidad y satisfacción en la distribución de las facultades naturales y contingencias de las condiciones sociales ${ }^{44}$.

Como hace ver Udi, por un lado, la noción de familia, y particularmente la idea de actuar como cabeza de familia para responder al problema de cómo repartir entre generaciones las cargas de construir y mantener una sociedad justa, permite incorporar en la posición de justicia original de Rawls el principio del ahorro justo, mediante un principio de justicia distributiva intergeneracional ${ }^{45}$.

Por otro, la familia en Rawls, además de ser necesaria para la reproducción sexual de los individuos, constituye una primera escuela para el desarrollo moral de los futuros integrantes de una sociedad bien ordenada: los niños y las niñas ${ }^{46}$. Sobre este punto, Rawls plantea que uno de los rasgos que definen a una sociedad bien ordenada es la estabilidad de la concepción de justicia que le rige. Para esto es necesario que los principios de justicia sean conocidos públicamente, reconocidos como justos, y causar suficiente convicción y disposición para comportarse de modo tal que se contribuya a mantener una sociedad justa 47 .

La apuntada por Rawls es una de las varias funciones sociales que tradicionalmente ha cumplido la familia: la de reproducción social. La familia, en su constante interrelación con los cambios sociales, juega

\footnotetext{
41 Íbid, 279 y 100.

42 Juliana Udi, "El valor de la familia en la teoría de la justicia de Rawls", 114.

43 Íbid, 118.

44 John Rawls, Teoría de la justicia, 461.

45 Íbid, 128; Juliana Udi, "El valor de la familia en la teoría de la justicia de Rawls", 119-121.

46 Íbid, 130.

47 John Rawls, Teoría de la justicia, 411-422.
} 
un papel nuclear en el diseño de cada sociedad y en la transmisión de valores, tradiciones, patrones culturales y reglas axiológicas. De hecho, esta función social ha propiciado el interés de los poderes políticos en la institución de la familia 48 .

Así las cosas, el valor de la familia en la transmisión de valores $\mathrm{o}$, en este caso, concepciones de justicia, se contradice con el lugar que, en ocasiones, Rawls le asigna como agrupación asociativa, y la valoración de las relaciones que ella entraña como privadas por ser meramente afectivas. En todo caso, esta justificación instrumental ha sido criticada por Okin desde lo sustantivo, por contradecir su propio concepto de justicia. La familia, al menos la de carácter patriarcal, difícilmente podría ser un espacio para inculcar las facultades morales que le preocupan a Rawls ${ }^{49}$.

Según Udi, el valor de la familia también puede ser justificado, desde la teoría de Rawls, como una estrategia normativa o deontológica, cuando se considera necesaria en virtud de derechos y libertades que la sustentan ${ }^{50}$, al ser la familia un fruto de la asociación voluntaria. En este sentido, la familia sería no solo condición sino efecto de una sociedad justa ${ }^{51}$. Una estrategia de justificación similar es la utilizada por Nussbaum al plantear que la pertenencia a una familia es manifestación del ejercicio de la libertad de asociación por parte de las personas adultas 52 .

Ahora bien, las respuestas que se han planteado, bien se dirigen a la consecución de determinados fines (una sociedad justa) o bien se centran en la satisfacción de los intereses de los padres y madres como personas adultas que ejercen su libertad de asociación. Los intereses de los niños y las niñas en sí no se avizoran en ellas directamente, sino para salvaguardar los intereses de la sociedad en la formación de futuras personas adultas convenientes para ella. Hay que reconocer, eso sí, que Rawls destaca el valor de la afectividad, los lazos de confianza y reafirmación de la autovalía en la relación paterno y maternofilial, particularmente en el ejercicio de los roles parentales, aspectos que son centrales en una socialización de los niños y las niñas correctamente entendida desde el punto de vista de sus derechos. No obstante, justifica la importancia de lo anterior en el desarrollo y entrenamiento de la moral de la autoridad y, a través de ello, lograr una sociedad bien ordenada en la que se transmita de forma estable el sentido de justicia, no así en los propios hijos e hijas ${ }^{53}$.

Si partimos de la base de que los niños y las niñas tienen derechos, como así lo han reconocido -casi unánimemente- los

\footnotetext{
48 Rosario Valpuesta, La disciplina constitucional de la familia en la experiencia europea, 53-56.

${ }^{49}$ Susan Moller Okin, Justice, gender and the family, 96-101

50 Juliana Udi, "El valor de la familia en la teoría de la justicia de Rawls", 112- 115.

51 Íbid, 112- 115

52 Martha Nussbaum, Las mujeres y el desarrollo humano, 362-363.

53 John Rawls, Teoría de la justicia, 411-422.
} 
Estados al ratificar la Convención sobre los Derechos del Niño, entonces sería coherente buscar alguna justificación de la familia centrada en sus intereses como sujetos actuales y completos. En este punto, considero que, desde la perspectiva de los efectos de los principios de justicia, también podría invocarse la función socializadora que la familia cumple, en general (admitiendo lamentables y no aisladas excepciones), para los niños y las niñas como sujetos de derechos. Esto es, constituir un espacio en el cual modelan su personalidad, desarrollan sus facultades y herramientas, aprenden las pautas de comportamiento que les forman para su madurez, y les otorga un ambiente propicio para el mayor equilibrio afectivo que facilita su desarrollo, además de promover su bienestar actual, brindándole estabilidad y seguridad física y emocional ${ }^{54}$.

\section{5.- EL LUGAR DE LA FAMILIA EN LA SOCIEDAD: ENTRE LA ESTRUCTURA BÁSICA Y LA AGRUPACIÓN AFILIATIVA}

Al definir el lugar que ocupa la familia en una sociedad, Rawls presenta notorias ambigüedades. Por un lado, la define como una gran institución social que forma parte de la estructura básica de la sociedad, y por otro, como una asociación voluntaria que no es parte de aquella. Que posea uno u otro valor no es baladí, pues, como aclara Spector, determinará si son coercitivamente aplicables en ella los principios de justicia o si será regida solamente por alguna concepción comprehensiva del bien ${ }^{55}$.

Rawls plantea que el objeto primario de la justicia es la estructura básica de la sociedad. Entiende por tal "el modo en que grandes instituciones sociales distribuyen los derechos $y$ deberes fundamentales y determinan la división de las ventajas provenientes de la cooperación social" 56 . Estas grandes instituciones sociales, mediante el establecimiento de ciertas reglas, "definen los derechos y deberes del hombre e influyen sobre sus perspectivas de vida, sobre lo que puede esperar hacer y sobre lo que haga" 57 . Es decir, sus efectos pueden generar desigualdades profundas para acceder a las oportunidades, incluso aquellas iniciales en la vida, lo que justificaría que sean el tema primario de justicia ${ }^{58}$. Aclara que no es la institución en sí justa o injusta, sino que lo es la forma en que su actividad es realizada y administrada ${ }^{59}$. Ofrece como ejemplos de estas grandes instituciones la constitución, la propiedad privada y la familia (pero no

\footnotetext{
54 Rosario Valpuesta, La disciplina constitucional de la familia en la experiencia europea, 87; David Archard, Children. Rights and childhood, 163.

55 Ezequiel Spector, "La familia en Rawls: ¿En qué sentido es parte de la estructura básica?" (2014), N 41, Isonomía, 98.

56 John Rawls, Teoría de la justicia, 20.

57 Íbid, 20 y 62.

58 Íbid, 20; John Rawls, Liberalismo político, 35-36.

59 John Rawls, Teoría de la justicia, 63.
} 
cualquiera: la monógama) ${ }^{60}$. La incorporación de la familia es coherente con sus efectos, pues ya vimos que la familia puede ser una importante fuente de desventaja.

En otros pasajes de su obra, parece alejarse de esta idea y remarcar la diferencia de lo público en oposición a lo privado, y dentro de lo último, el ámbito familiar, para remarcar que su liberalismo político no preconiza ninguna idea comprehensiva del bien ${ }^{61}$. Cuando asume dicha postura, Rawls parece acercar a la familia más a las agrupaciones voluntarias, tales como las iglesias y universidades, que a la estructura básica de la sociedad. Incluso, pone a lo familiar como ejemplo de lo no político, junto con lo asociativo y lo personal62. Aclara que ideas del bien, como las asociadas a "las virtudes apropiadas para los papeles en la vida en familia y a las relaciones entre individuos"63, no pueden formar parte de la concepción política de la justicia como sí podrían serlo las ideas políticas, ya que estas últimas - a diferencia de las primeras, habría que entender - "nos permite suponer que la comparten los ciudadanos y no depende de ninguna doctrina comprensiva en particular"64. Así las cosas, la autonomía de la organización familiar interna corresponde a visiones comprehensivas que, por tanto, quedarían fuera del margen de acción de un Estado democrático en una sociedad pluralista.

Ante esta ambivalencia, Spector plantea entender que las instituciones voluntarias como la universidad, la iglesia, y especialmente - la familia, tienen una naturaleza compleja. Esto quiere decir que: i) la familia no será siempre parte de la estructura básica; y ii) algunas (o, más bien, varias) veces y en determinados aspectos sí lo es, según los efectos que produzcan en la posición de las personas. En esto último se debería tener especialmente en cuenta que la falta de aplicación de los principios de justicia devendría en una desventaja 65 .

\section{6.- LO DOMÉSTICO, ¿UNA BARRERA INFRANQUEABLE PARA LOS DERECHOS DE LOS NIÑOS Y LAS NIÑAS?}

Según se ha venido diciendo, Rawls, especialmente en el Liberalismo Político, no aplica directamente su concepción de justicia a los problemas que se suscitan en las relaciones familiares. Incluso, algunos pasajes de su obra sugieren lo contrario: que la justicia como equidad se aplica solo al ámbito de lo político, que se distingue de lo familiar junto con lo personal y asociativo. La esfera familiar, a

60 Íbid, 20; John Rawls, "The idea of public reason revisited" (1997), vol. 64, N 3, The University of Chicago Law Review, 788.

61 John Rawls, Liberalismo político, 35-38, 140 y 190.

62 Íbid, 140.

63 Íbid, 188

64 Íbid, 188.

65 Ezequiel Spector, "La familia en Rawls: ¿En qué sentido es parte de la estructura básica?", 110. 
diferencia de la política, indica Rawls, pertenece al ámbito de los afectos, y se rige por valores morales propios ${ }^{66}$. Esto, porque el liberalismo busca distinguir el punto de vista público de aquellos que no lo son, y ser imparcial entre los distintos puntos de vista de las doctrinas comprehensivas razonables ${ }^{67}$.

No obstante, Rawls intenta dejar en claro que ello no quiere decir que queden fuera de la esfera de la justicia, puesto que sus principios igualmente les son aplicables como parte integrante de la estructura social básica. Una concepción de justicia, en coherencia con sus propios principios de justicia, puede alentar, desalentar o incluso prohibir determinados puntos de vista que entren en conflicto con ellos ${ }^{68}$. Así, por ejemplo, en una sociedad en donde la tolerancia y el respeto mutuo son un valor, su régimen constitucional podría válidamente tomar ciertas medidas para reforzarlo, como discriminación por determinados motivos (de manera coherente con la libertad de pensamiento y expresión), sin ser por ello parcial ${ }^{69}$.

Bajo esta forma, Rawls estaría proponiendo límites externos a la familia, así como a otras asociaciones voluntarias, que no afectan su gobierno interno. $O$ al menos no directamente, ya que la familia y sus integrantes se encuentran insertos en una sociedad que está, a la vez, gobernada por instituciones básicas ${ }^{70}$.

Como apunta Nussbaum, estos límites externos no serían suficientes para abordar la situación de desventaja de todas las personas. Para ella, finalmente, esta teoría sería tolerante con prácticas que, si bien son indeseables por provocar esa desventaja, no ameritarían intervención por no ser una violación de los principios de justicia básica, como lo sería, por ejemplo, que la persona amenazada no pudiera abandonar la familia o estuviera en peligro su integridad física ${ }^{71}$. Para ella, tampoco queda claro qué diferencia concretamente la aplicación de los principios de justicia a la familia de forma indirecta como parte de la estructura básica, de hacerlo de forma directa como una institución social que forma parte de dicha estructura ${ }^{72}$.

Considero que es correcto entender, siguiendo a Nussbaum, que el Derecho no solamente interviene mediante una acción directa en la familia cuando, de acuerdo a la concepción de justicia, lo amerita, sino que la constituye. La familia no es una unidad pre-política ni prejurídica, así como tampoco es neutra, puesto que su existencia, término, consecuencias, así como el límite y contenido de sus relaciones, están finalmente insertos en un sistema jurídico que la crea y regula (incluso al decidir mantener el status quo). Esto es importante,

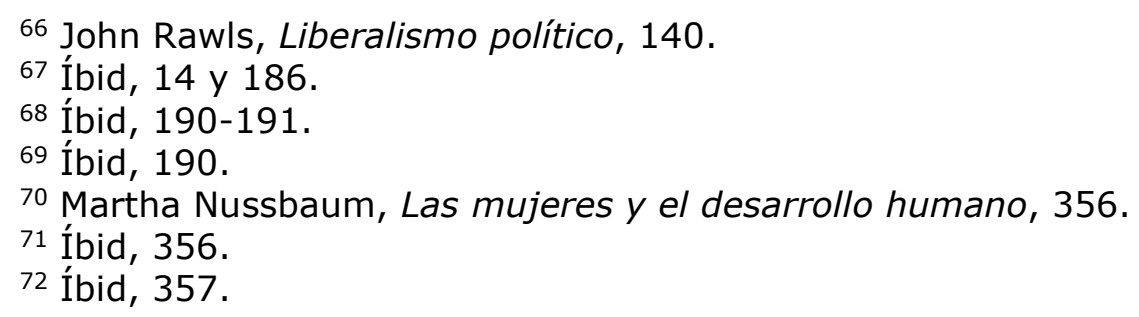


puesto que nos obliga a entender a la familia no como un hecho dado y natural, sino como un fenómeno social y también jurídico, que, por tanto, es ya un asunto público ${ }^{73}$. En este sentido, la elección sobre no intervenir en determinados modos de vida al interior de la familia no implica necesariamente una neutralidad pura. Por otro lado, los límites no son solo externos, sino que también la constituyen ${ }^{74}$. A partir de su crítica, Nussbaum propone centrarse en las capacidades y libertades de cada persona (en vez de en la familia en sí como grupo), y no asumir alguna agrupación afiliativa como prioritaria o central ${ }^{75}$.

Me parece que, adoptando algunas ideas del enfoque de Nussbaum, específicamente la necesidad de centrarnos en cada persona como un fin, admitir que los límites jurídicos no son solamente externos a la familia y dotar de una interpretación necesariamente contextual, podemos interpretar la teoría de Rawls desde un enfoque de derechos humanos de los niños y las niñas en la resolución de los problemas dentro de la familia.

Indica Rawls en The Idea of Public Reason Revisited que, en una sociedad democrática, el Estado estará probablemente interesado en regular ordenadamente las instituciones que son necesarias para que la sociedad se reproduzca. Esto incluiría ámbitos como la familia, en aspectos como la crianza y educación de los niños y las niñas. Pero no tendría un interés legítimo en regular ciertos aspectos como un modelo particular de familia o las relaciones entre sus miembros, puesto que, como se ha dicho, implicaría comprometerse con cierta doctrina moral comprehensiva. Lo anterior, salvo que estos afecten de algún modo la reproducción de la sociedad en el tiempo. Considero que esta interpretación debe ser efectuada necesariamente de manera contextual, de manera que admitirá distintas excepciones que puedan afectar la transmisión de los valores y principios de la sociedad ${ }^{76}$. Un límite a dicha libertad debería ser la afectación de derechos fundamentales de sus integrantes, particularmente de los niños y las niñas.

En efecto, Rawls recuerda que los miembros de la familia son ciudadanos titulares de derechos antes que integrantes de un grupo ${ }^{77}$. Respecto de los niños y las niñas, probablemente Rawls, al igual que Nussbaum, justificarían lo anterior en su calidad de futuros ciudadanos. Se deberá enriquecer la teoría, entonces, con un enfoque de derechos de los niños y las niñas, para atender realmente a su desventaja actual por el valor que tienen como personas actuales.

\footnotetext{
73 Íbid, 361-362. "Rawls parece mantener, pues, en forma análoga, una distinción entre acción e inacción del estado que sugiere que el estado no está actuando cuando no interfiere en el modelo tradicional de familia, mientras que estaría actuando si intentara cambiar los modelos de gobierno de la familia". Íbid, 363.

74 Ibídem.

75 Íbid, 368.

76 John Rawls, "The idea of public reason revisited", 779.

77 Íbid, 791.
} 
Así las cosas, asumiendo el carácter conformador del Derecho en la familia, sería apropiada su regulación ya no exclusivamente mediante el derecho privado, particularmente el derecho de familia y civil, sino también mediante derecho público, particularmente desde la óptica de los derechos humanos y su reconocimiento en la Constitución. Esto permitirá ajustar las relaciones de familia relevantes jurídicamente conforme los principios que informan la organización política y social de nuestra sociedad ${ }^{78}$, de acuerdo a su propia concepción de justicia.

En el campo de la familia, los derechos humanos, a diferencia de su noción clásica como límites frente al poder estatal, se convierten en garantías para que las relaciones recíprocas que se entretejen horizontalmente en la ciudadanía, particularmente en la esfera familiar, se rijan también por principios y valores trascendentales como la igualdad, la libertad y la dignidad. Esta es una forma de proteger a los miembros como personas titulares de derechos, con preminencia sobre la estabilidad de la familia como tal79. Para eso, los textos constitucionales otorgan garantías y mecanismos de resistencia que aseguran, al menos con más fuerza que una ley corriente, su aplicación.

La introducción de las ideas de justicia, particularmente a través del discurso de los derechos, en el ámbito de las relaciones familiares no implica negar a las familias y a los individuos que la componen un ámbito propio de autonomía y realización, ni imponer cierta doctrina comprensiva. Contrariamente, como hace ver Valpuesta, el foco sobre cada integrante de la familia como persona y titular de derechos lleva a un más amplio reconocimiento de la autonomía en las relaciones y la vida familiar ${ }^{80}$ y entrega más condiciones para que las personas puedan llevar a cabo sus planes de vida en igualdad.

\section{7.- CONCLUSIONES}

En Rawls es posible observar de forma clara la tensión existente desde el liberalismo en relación con la familia. Por un lado, la familia significa una esfera privada de afectividad, elección personal y desarrollo de planes de vida, que debe poder desenvolverse libremente $y$, en consecuencia, permanecer libre de injerencias sociales $y$ estatales. Por otro lado, Rawls reconoce la profunda influencia de la familia en el desarrollo humano, el carácter inmerecido de las condiciones que nos otorga el nacimiento en cierta familia, así como también su papel de condicionante en el acceso a la igualdad de oportunidades. De esta forma, la familia se planta de frente a los principios de justicia. Probablemente por esa tensión, Rawls presenta

78 Rosario Valpuesta, La disciplina constitucional de la familia en la experiencia europea, 113.

79 Íbid, 248.

80 Íbid, 211. 
algunas ambivalencias en su obra, que, no obstante, han suscitado provechosas revisiones, reflexiones y críticas que nos permiten poner luz sobre esta problemática.

La familia ha sido criticada desde distintas perspectivas por ser una potencial fuente de desventaja, especialmente para quienes se encuentran en posición de subordinación en relación al pater. Rawls, por su parte, también desidealiza la familia al aceptar que puede resultar siendo una fuerte condicionadora de desventajas, lo que iría en contra de los principios de justicia que propone. Pese a eso, la familia, para Rawls, ocupa un rol trascendental que justifica su existencia, pues funciona como transmisora de su concepción de justicia; aunque deja de lado la función que ella cumple o no en relación con la niñez propiamente tal. Se debate, no obstante, en considerar si es parte o no de la estructura social básica. Pero, sea directamente como institución que conforma la estructura social básica, sea como grupo inserto en ella, los principios de justicia le son aplicables, al menos indirectamente en el último caso.

Si bien podemos poner en duda el carácter de indirecto de esa intervención, atendido que el Derecho configura a la familia, una correcta aplicación de esta idea, incorporando a los niños y las niñas como sujetos de derechos humanos que funcionan como límites en las relaciones intersubjetivas, permite trabajar sobre su desventaja, sin afectar por ello la autonomía de la vida familiar. En definitiva, pareciera ser que la ambivalencia más relevante la presenta el largo debate acerca de lo público y lo privado, específicamente qué se entiende por privado, cuáles son sus consecuencias y qué se entiende por intervenir en dicha esfera.

\section{8.- BIBLIOGRAFÍA}

Archard, David, Children. Rights and childhood (2० ed, Routledge, Oxon, 2004).

Archard, David, Children, family and the State (Routledge revivals, Londres y Nueva York, 2018).

Fanlo, Isabel [comp.], Derecho de los niños. Una contribución teórica (Biblioteca de Ética, Filosofía del Derecho y Política, México DF, 2004).

Farson, Richard, Birthrights (Macmillan Publishing, Nueva York, 1974).

Filmer, Robert, Pathriarca and other political works of Sir Robert Filmer (Basil Blackwell Oxford, Oxford, 1949).

Garzón Ernesto, "Desde la 'Modesta Propuesta' de J. Swift hasta Las 'Casas de Engorde': Algunas consideraciones acerca de los derechos de los niños" (1994), Doxa, 15, 731.

Giddens, Anthony, Sociología (traducido por: Muñoz, F., $6^{\circ}$ ed., Alianza Editorial, Madrid, 2014).

Holt, John, Escape from childhood. The needs and rights of children (Penguin Books, Hardmondsworth, 1975). 
Locke, John, Segundo tratado sobre el gobierno civil. Un ensayo acerca del verdadero origen, alcance y fin del Gobierno Civil (traducido por: Mellizo, C., Tecnos, Madrid, 2006).

Locke John, Ensayo sobre el gobierno civil ( $1^{\circ}$ ed. electrónica, Letras para volar, Guadalajara, 2015).

MacCormick, Neil, "Los derechos de los niños: un test para las teorías de los derechos", en: Fanlo, Isabel [comp.], Derecho de los niños. Una contribución teórica (Biblioteca de Ética, Filosofía del Derecho y Política, México DF, 2004), 61.

Nussbaum, Martha, Las mujeres y el desarrollo humano (traducido por: Bernet, R., $2^{\circ}$ ed., Herder, Barcelona, 2017).

Okin, Susan Moller Justice, gender and the family (Basic Books Inc., Nueva York, 1989).

Rawls, John, Liberalismo político (traducido por: Madero, S., Fondo de Cultura Económica, México DF, 1995).

Rawls, John, Teoría de la justicia (traducido por: González, M., Fondo de cultura económica, México DF, 1997).

Rawls, John, "The idea of public reason revisited" (1997), vol. 64, Nº 3, The University of Chicago Law Review, 765.

Pateman, Carole, El contrato sexual (traducido por: Femenías, María Luisa, primera edición, Anthropos, México DF, 1995).

Spector, Ezequiel, "La familia en Rawls: ¿En qué sentido es parte de la estructura básica?" (2014), № 41, Isonomía, 97.

Turégano, Isabel, "La dicotomía público/privado y el liberalismo político de J. Rawls" (2001), N²4, Doxa, 319.

Udi, Juliana, "El valor de la familia en la teoría de la justicia de Rawls" (2017), No 47, Isonomía, 109.

Valdés, Ximena, La vida en común. Familia y vida privada en Chile y el medio rural en la segunda mitad del siglo XX ( $1^{\circ}$ ed., Lom Ediciones, Santiago, 2007).

Valpuesta, Rosario, La disciplina constitucional de la familia en la experiencia europea (Tirant lo Blanch, Valencia, 2012). 